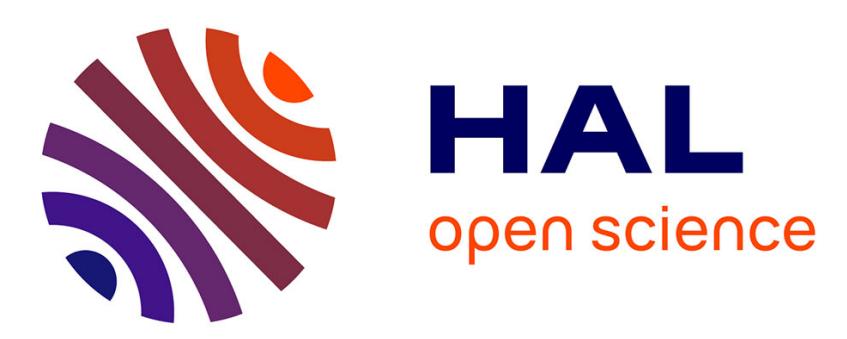

\title{
Modes de structuration des contenus perceptifs visuels
}

\author{
Elisabeth Pacherie
}

\section{To cite this version:}

Elisabeth Pacherie. Modes de structuration des contenus perceptifs visuels. J. Bouveresse; J.-J. Rosat. Philosophies de la perception, Odile Jacob, pp.263-289, 2003. ijn_00778252

\section{HAL Id: ijn_00778252 \\ https://hal.science/ijn_00778252}

Submitted on 19 Jan 2013

HAL is a multi-disciplinary open access archive for the deposit and dissemination of scientific research documents, whether they are published or not. The documents may come from teaching and research institutions in France or abroad, or from public or private research centers.
L'archive ouverte pluridisciplinaire HAL, est destinée au dépôt et à la diffusion de documents scientifiques de niveau recherche, publiés ou non, émanant des établissements d'enseignement et de recherche français ou étrangers, des laboratoires publics ou privés. 
Pacherie, E. 2003. Modes de structuration des contenus perceptifs visuels. Philosophies de la perception, sous la dir. de J. Bouveresse \& J.-J. Rosat, Paris: Éditions Odile Jacob, pp. 263-289.

\title{
MODES DE STRUCTURATION DES CONTENUS PERCEPTIFS VISUELS
}

\author{
Élisabeth Pacherie \\ Institut Jean Nicod \\ CNRS, Paris
}

\section{Introduction}

Le débat sur la nature des contenus perceptifs occupe depuis une vingtaine d'années le devant de la scène en philosophie de la perception. Ce débat oppose tenants d'une approche conceptualiste (McDowell, 1994; Sedivy, 1996; Brewer, 1999) qui soutiennent que les contenus perceptifs sont entièrement conceptuels et partisans d'une approche nonconceptualiste (Evans, 1982; Peacocke, 1992, 1998, 2001; Crane, 1992; Dretske, 1995; Bermúdez, 1998) qui affirment, à l'inverse, que les contenus perceptifs ne sauraient être entièrement conceptuels même s'ils peuvent être en partie conceptualisés. La défense d'une position non-conceptualiste comporte deux volets. Le premier est essentiellement négatif. Il s'agit de montrer que les expériences perceptives ont, relativement aux croyances perceptives ou jugements de perception, un certain nombre de caractéristiques distinctives dont on ne saurait rendre compte si l'on postule que leur contenu est de type purement conceptuel. Il s'agit donc d'établir la thèse négative selon laquelle le contenu de la perception ne saurait être purement conceptuel. Le second volet de cette défense consiste à proposer une caractérisation positive du contenu des expériences perceptives et ainsi de la notion de contenu nonconceptuel. Il s'agit en premier lieu de montrer que la notion de contenu non-conceptuel n'est pas incohérente, autrement dit, qu'il n'est pas contradictoire de soutenir qu'à certains niveaux au moins, les contenus perceptifs ne sont pas conceptuels mais sont néanmoins intentionnels 
ou représentationnels ${ }^{1}$. Il s'agit en outre de montrer que la notion de contenu non-conceptuel a une utilité explicative et notamment qu'elle permet de rendre compte de certaines caractéristiques de nos expériences perceptives et de certains des rôles joués par la perception dans l'économie cognitive.

Je me rangerai ici aux côtés des non-conceptualistes. Je tenterai d'apporter une contribution au volet positif de leur démarche et avancerai quelques propositions sur les modes de structuration des contenus perceptifs qui me paraissent susceptibles d'éclairer certains aspects de l'interface entre la perception et d'autres dimensions de la cognition. Je rappellerai tout d'abord brièvement les principaux arguments négatifs qui ont été proposés dans la littérature à l'encontre de l'idée que le contenu de la perception est entièrement conceptuel. Je préciserai ensuite les enjeux d'une caractérisation positive du contenu nonconceptuel. J'examinerai certaines propositions de Christopher Peacocke et notamment les notions de contenu-scénario et de contenu protopropositionnel qu'il a introduites. Mon objectif principal sera de préciser la notion de contenu protopropositionnel au-delà de l'esquisse proposée par Peacocke. Pour ce faire je m'appuierai en particulier sur certains travaux empiriques sur l'attention visuelle et ses déficits et sur la segmentation des scènes visuelles.

\section{Arguments négatifs}

Les principaux arguments avancés par les non-conceptualistes en faveur de la thèse négative selon laquelle les contenus perceptifs ne sauraient être entièrement conceptuels exploitent plusieurs caractéristiques spécifiques des expériences perceptives: leur indépendance vis-à-vis des croyances, leur finesse de grain, leur richesse et leur caractère perspectival. Je rappelle brièvement ces arguments.

\subsection{Indépendance vis-à-vis des croyances}

Un certain nombre de défenseurs de l'approche non-conceptualiste (Evans, 1982; Dretske, 1969; Peacocke, 1992; Crane, 1992) ont fait remarquer que l'expérience perceptive manifeste une forme d'indépendance vis-à-vis des croyances. Nos états perceptifs peuvent avoir leur contenu propre indépendamment du fait que nous ayons ou non des croyances

\footnotetext{
${ }^{1}$ J'utiliserai tout au long de ce texte les notions d'état intentionnel, d'état représentationnel ou d'état doté d'un contenu de manière interchangeable. Je précise qu'en parlant des états perceptifs comme d'états représentationnels je n'entends nullement marquer une adhésion à la théorie dite représentationnaliste ou indirecte de la perception selon laquelle dans la perception nous aurions directement conscience d'une représentation et seulement indirectement du monde à travers cette représentation.
} 
correspondantes. Les illusions perceptives, comme l'illusion de Müller-Lyer, illustrent cette indépendance. Dans l'illusion de Müller-Lyer, on présente au sujet deux lignes de même longueur mais dont l'une se termine par des flèches pointant vers l'extérieur, l'autre par des flèches pointant vers l'intérieur. Il est bien connu que même si le sujet sait que les lignes sont de même longueur, il ne peut s'empêcher de voir l'une comme plus longue que l'autre. L'existence de cette forme d'indépendance vis-à-vis des croyances montre que nous avons affaire à des types d'attitudes différents vis-à-vis d'un certain contenu; mais elle ne permet pas de conclure au caractère non-conceptuel des contenus perceptifs.

Il existe toutefois une notion plus forte d'indépendance vis-à-vis des croyances qui semble nous conduire à cette conclusion. Cette notion forte d'indépendance a été introduite par Dretske (1969) dans le cadre de sa discussion de la distinction entre vision épistémique et vision non-épistémique. Dretske caractérise la vision non-épistémique comme étant dépourvue de contenu doxastique. Il soutient qu'il s'agit d'une capacité visuelle primitive qui ne requiert pas la possession de croyances ou d'ensembles particuliers de croyances de la part de l'agent. Autrement dit, quelle que soit la proposition P, 'X voit D' (au sens nonépistémique) n'implique pas logiquement $X$ croit que $P$. On peut ainsi, voir un tigre au sens non-épistémique sans croire qu'il s'agit d'un tigre, d'un animal ou même d'une entité physique. Il semble que s'il existe bien une forme de vision non-épistémique indépendante des croyances en ce sens fort, on doive conclure que son contenu ne saurait être conceptuel. La prémisse supplémentaire qui permet d'aboutir à cette conclusion est que la possession d'un concept implique essentiellement certaines croyances, notamment des croyances sur ses conditions d'application. Si la vision non-épistémique est en principe indépendante de toute croyance particulière, elle l'est aussi des croyances constitutives de la possession de concepts et donc son contenu ne saurait être conceptuel. Cet argument en faveur du caractère nonconceptuel du contenu perceptif reste toutefois conditionnel. Si, comme l'affirme Dretske, certains états perceptifs sont indépendants des croyances au sens fort, alors ils ne sauraient avoir de contenu conceptuel. Mais dire que ces états perceptifs n'ont pas de contenu conceptuel n'est pas encore dire qu'ils ont un contenu non-conceptuel; une autre possibilité demeure qui est que ces états n'aient pas à proprement parler de contenu intentionnel ou représentationnel. La conclusion conditionnelle à laquelle nous sommes conduits est donc la suivante: si le voir non-épistémique a un contenu intentionnel, alors ce contenu ne saurait être conceptuel. Pour aboutir à la conclusion non-conceptualiste désirée, il faut encore montrer que le voir non-épistémique a un contenu intentionnel. Une telle démonstration suppose que 
l'on soit en mesure de donner une caractérisation positive de ce qu'est la vision nonépistémique.

\subsection{Finesse de grain}

L'argument de la finesse de grain (Evans, 1982; Peacocke, 1992, 2001) exploite l'idée que nous pouvons normalement opérer des discriminations perceptives beaucoup plus fines que les discriminations autorisées par les concepts observationnels que nous possédons. Ainsi, nous pouvons discriminer plusieurs millions de nuances de couleur alors que nous ne possédons au mieux que quelques centaines de concepts de couleur. Il en va de même des formes, des textures ou des grandeurs spatiales. La conclusion qu'en tirent les nonconceptualistes est que, puisque la gamme de contenus discriminables que peuvent avoir nos expériences perceptives excède largement la gamme des discriminations permises par les concepts appartenant au répertoire d'un sujet, les contenus perceptifs doivent être nonconceptuels.

McDowell (1994) et Brewer (1999) ont opposé une réplique conceptualiste à cet argument. Ils ne contestent nullement que l'expérience perceptive ait une grande finesse de grain mais ils soutiennent que l'on peut parfaitement l'appréhender au moyen de concepts. Le reproche qu'ils adressent aux non-conceptualistes est d'adopter une conception indûment restrictive des concepts, considérés comme essentiellement descriptifs et indépendants du contexte. Selon McDowell et Brewer, cette restriction n'a pas lieu d'être. Si on ne peut appréhender toute la finesse de grain des expériences perceptives au moyen des seuls concepts descriptifs, on peut parfaitement le faire au moyen de concepts démonstratifs, tels que "cette couleur", "cette nuance", "ce rouge" ou même "ceci" employés en présence d'un échantillon de couleur.

Cette réplique conceptualiste s'expose elle-même à plusieurs objections. On peut notamment se demander à quelles conditions penser à une nuance de couleur comme "cette nuance" constitue une manifestation authentique d'une capacité conceptuelle. Il semble, comme le reconnaît McDowell, que la maîtrise d'un concept suppose la capacité à identifier et réidentifier à travers le temps, fût-ce pour une brève période, des échantillons d'une même nuance et non simplement à discriminer deux échantillons de couleur présentés simultanément. Or, d'abondantes données expérimentales montrent que nos capacités de discrimination perceptive dépassent largement nos capacités d'identification ou de reconnaissance (Burns et Ward, 1977; Halsey et Chapanis, 1951; Hardin, 1988; Hurvich; 1981). A l'évidence, on ne peut reconnaître que ce que l'on a pu mémoriser. Des études de 
psychophysique et de psychologie de la perception montrent que la mémoire perceptive est limitée et que son grain est beaucoup plus grossier que le grain correspondant aux seuils de discrimination perceptive. Il s'ensuit que si la possession d'un concept suppose une certaine capacité de reconnaissance et d'identification, la finesse de grain maximale de nos concepts perceptifs correspondra à la finesse de grain maximale de l'encodage en mémoire perceptive. Les conceptualistes qui font appel à des concepts démonstratifs sont donc placés devant un dilemme. Ou bien ils admettent que ces concepts démonstratifs doivent satisfaire à une condition de reconnaissance et dans ce cas leur finesse de grain sera inférieure à celle dont témoignent nos capacités de discrimination perceptive, ce qui revient à admettre que l'on ne peut appréhender toute la finesse de grain des expériences perceptives au moyen de concepts, fussent-ils démonstratifs. Ou bien, ils renoncent à la condition de reconnaissance, mais, ce faisant, se heurtent à d'autres difficultés. Selon le Critère de Différence, d'inspiration frégéenne, deux concepts sont différents si un sujet peut rationnellement adopter des attitudes différentes à l'égard de deux pensées qui ne diffèrent qu'en ce que la première contient l'un de ces concepts et la seconde l'autre. Supposons que l'on présente successivement à un sujet deux échantillons (ou le même échantillon) de la même nuance de couleur, $a_{1}$ et $a_{2}$. Il est naturel de penser que les concepts démonstratifs fondés sur la perception de ces échantillons devraient être les mêmes, puisque ou bien il s'agit du même échantillon ou bien ces échantillons sont exactement de la même couleur. Toutefois, il est toujours possible pour un sujet rationnel de croire que tout ce qui est de cette couleur $r_{a 1}$ est de cette couleur ${ }_{a 1}$, tout en doutant que tout ce qui est de cette couleur $r_{a 1}$ soit de cette couleur ${ }_{a 2}$. Pour ce qu'il en sait sur la base de son expérience perceptive, il est possible que $a_{1}$ et $a_{2}$, perçus simultanément, soient discriminables. Il s'agit d'une possibilité épistémique cohérente, ce qui implique, selon le Critère de Différence, que 'cette couleur $a_{a 1}$ ' et cette couleur $a_{a 2}$ ' expriment des concepts différents. Si nous acceptons le Critère de Différence pour les concepts, il apparaît impossible de saisir le même concept démonstratif à travers la perception d'échantillons de la même nuance en différentes occasions. Il y a donc un nombre infini de concepts démonstratifs distincts pour une même nuance, autant que d'expériences perceptives de cette nuance. Les conceptualistes doivent alors admettrent ou bien qu'il existe dans les contenus de perception des différences conceptuelles qui ne correspondent à aucune différence phénoménologique ou bien qu'ils découpent trop finement le monde phénoménal. En bref, donc, ou bien l'approche conceptualiste n'est pas assez fine ou bien elle l'est trop. ${ }^{2}$

\footnotetext{
${ }^{2}$ Pour une analyse plus détaillée de ce débat entre conceptualistes et non-conceptualistes sur la finesse de grain,
} 
Il existe un second type d'argument exploitant la finesse de grain de l'expérience perceptive. La logique de cet argument est légèrement différente car il met en jeu non pas des discriminations entre propriétés perceptives mais différentes manières de percevoir une même chose. Peacocke (1992) illustre cette possibilité à travers le cas d'une forme que l'on peut percevoir soit comme un losange régulier soit comme un carré posé sur la pointe. Deux sujets peuvent percevoir une forme différemment et ceci même s'ils utilisent le même concept démonstratif, 'cette forme'. Selon l'explication proposée par Peacocke, les sujets sont sensibles à des aspects différents de la forme. Celui qui la perçoit comme carré est sensible aux symétries par rapports aux bissectrices des côtés tandis que celui qui la perçoit comme losange est sensible aux symétries par rapport aux bissectrices des angles. Le conceptualiste pourrait soutenir que remarquer ou prêter attention à certaines symétries revient à les conceptualiser démonstrativement et donc que la différence entre ces deux manières d'appréhender une forme est une différence conceptuelle. Mais cette conclusion repose sur la prémisse selon laquelle la sélection attentionnelle de certaines informations perceptives est une forme de conceptualisation. On doit toutefois distinguer deux formes de sélection attentionnelle. La sélection attentionnelle activement dirigée vers un aspect donné d'une scène visuelle et la sélection attentionnelle passive où l'attention est attirée par un aspect saillant de la scène visuelle. On peut éventuellement concéder au conceptualiste que la sélection active constitue une forme de conceptualisation démonstrative, mais douter qu'il en aille de même de la sélection attentionnelle passive. Il paraît au contraire plus plausible de soutenir que c'est le fait que l'attention ait été passivement attirée par certains aspects saillants d'une scène visuelle rend possible une conceptualisation démonstrative. Or, ceci revient à dire que la conceptualisation démonstrative exploite une information visuelle préalablement structurée, en d'autres termes que cette forme de conceptualisation exploite un niveau préalable de contenu perceptif où l'information visuelle est déjà structurée.

\subsection{Richesse informationnelle et caractère perspectival de l'expérience perceptive}

L'argument de l'indépendance vis-à-vis des croyances et l'argument de la finesse de grain sous ses deux formes visent à montrer que les primitives représentationnelles du contenu perceptives ne sont pas des concepts. L'argument de la richesse informationnelle vise, quant à

voir Dokic et Pacherie (2001) 
lui, à montrer que la structure des représentations perceptives n'est pas de même type que celle des représentations conceptuelles propositionnelles.

On remarquera tout d'abord que richesse informationnelle et finesse de grain sont des caractéristiques certes liées mais néanmoins distinctes de l'expérience perceptive. La finesse de grain renvoie au fait qu'il existe de nombreuses dimensions - couleur, forme, taille, direction, etc. - telles que n'importe quelle valeur sur ces dimensions peut entrer dans le contenu de l'expérience perceptive. La richesse informationnelle renvoie au fait que l'expérience perceptive est en général simultanément porteuse d'informations sur les valeurs de nombreuses dimensions. La distinction entre finesse de grain et richesse informationnelle est parfois obscurcie du fait d'un usage indifférencié de l'expression "caractère analogique du contenu perceptif", tantôt utilisée pour faire référence à l'une de ces propriétés, tantôt à l'autre.

En quoi la richesse informationnelle du contenu perceptif suggère-t-elle que son format n'est pas le format standard des représentations conceptuelles? Il semble que lorsqu'un sujet voit un objet dans des conditions normales, il ne puisse le voir sans voir à la fois sa forme, sa taille, sa couleur, sa texture, sa localisation spatiale et ainsi de suite. Une représentation de format conceptuelle peut fort bien, en revanche, encoder une propriété de l'objet sans encoder les autres. Ce n'est pas dire qu'une représentation conceptuelle ne puisse être informationnellement riche. A l'évidence, on peut, par un moyen aussi simple que la conjonction, construire des représentations conceptuelles aussi riches qu'on le souhaite. La différence tient plutôt à ce que la richesse informationnelle n'est pas une caractéristique obligatoire des représentations conceptuelles. C'est, au contraire, une vertu de ces représentations que de nous permettre de faire abstraction d'informations non pertinentes pour nos besoins. C'est donc le caractère obligatoire de la richesse informationnelle qui fait la spécificité des contenus perceptifs par rapport à des contenus conceptuels tels que, par exemple, les contenus de croyances. ${ }^{3}$

Cette richesse informationnelle est associée à une autre caractéristique des expériences perceptives qui est d'avoir un caractère perspectival. Pour tout trait d'une scène visuelle qui est représenté perceptivement, ce qu'encode le contenu perceptif n'est pas simplement la présence de ce trait mais aussi sa position dans l'espace égocentrique du sujet. Il semble donc qu'il y ait une solidarité entre la richesse informationnelle et le caractère perspectival des contenus perceptifs. Chaque trait représenté perceptivement est représenté comme occupant une

\footnotetext{
${ }^{3}$ On notera que l'argument de la richesse informationnelle concerne la structure des contenus perceptifs et non pas la nature de ses primitives représentationnelles puisqu'il ne permet pas à lui seul d'exclure que ces éléments représentationnels soient de type conceptuel.
} 
certaine position dans le champ visuel et inversement chaque position dans le champ visuel est caractérisée par la présence ou l'absence de certains traits. En particulier, il n'existe pas de trou dans le champ visuel. Ceci suggère que le mode d'organisation de l'information perceptive visuelle est essentiellement spatial ou cartographique. Le caractère obligatoire de la richesse informationnelle va ainsi de pair avec le caractère obligatoire d'une organisation spatiale égocentrique de l'information. Cette seconde caractéristique comme la première distingue les contenus perceptifs visuels des contenus conceptuels ordinaires. Il est certes possible de construire des représentations conceptuelles qui incluent des informations sur la position spatiale égocentrique des traits et propriétés représentés. Mais la représentation de l'information spatiale est en général optionnelle dans les représentations conceptuelles et les concepts spatiaux égocentriques ne jouissent d'aucun privilège particulier relativement à d'autres catégories de concepts.

Les arguments qui viennent d'être rappelés sont pour l'essentiel des arguments négatifs visant à montrer que les contenus perceptifs ont des caractéristiques spécifiques qui les distinguent des contenus conceptuels. L'argument de l'indépendance vis-à-vis des croyances, comme l'argument de la finesse de grain suggèrent que certains au moins des constituants représentationnels des contenus perceptifs ne sont pas des concepts. La richesse informationnelle et le caractère perspectival des expériences perceptives donnent à penser que les représentations perceptives ont un mode de structuration spécifique qui les distingue des représentations conceptuelles propositionnelles. Comme je l'ai souligné dans l'introduction, il est également nécessaire de proposer une caractérisation positive du contenu des expériences perceptives. Cette caractérisation devra satisfaire à trois types de contraintes: (i) montrer que l'expérience perceptive a bien un contenu intentionnel, même si celui-ci est non-conceptuel; (ii) rendre compte adéquatement de la phénoménologie de l'expérience perceptive; et (iii) montrer comment la perception peut s'intégrer à la cognition.

\section{Critères d'intentionnalité}

L'une des tâches des défenseurs d'une approche non-conceptualiste des contenus de perception consiste à montrer que la notion de contenu non-conceptuel est cohérente; autrement dit, que c'est bien d'une forme de contenu intentionnel qu'il s'agit. Pour ce faire, il importe d'énoncer les critères qu'un état devrait satisfaire pour que l'on puisse dire à bon droit qu'il s'agit d'un état représentationnel ou encore d'un état doté d'un contenu intentionnel. Je m'inspirerai sur ce point des propositions avancées par Bermúdez (1998). 
Le premier critère considéré par Bermúdez, à la suite de Peacocke, est qu'un état ne peut être considéré comme représentationnel que pour autant qu'il a des conditions de correction. On ne peut dire d'un état qu'il représente le monde d'une certaine façon que pour autant qu'il est possible d'énoncer une condition ou un ensemble de conditions sous lesquelles cette représentation est une représentation correcte du monde, le contenu de cet état étant défini par ces conditions. Ce premier critère est nécessaire mais non suffisant. En particulier, il ne permet pas de distinguer les états authentiquement représentationnels de ce que l'on peut appeler à la suite de Dretske $(1981,1995)$ les états informationnels. Dans la terminologie de Dretske, un état est dit informationnel ou porteur d'information sur un autre état si et seulement s'il existe une forme de covariation nomique entre les deux états. C'est le cas par exemple de la relation entre le nombre de cernes du tronc d'un arbre et l'âge de cet arbre. Un état informationnel a, comme un état représentationnel, des conditions de correction, mais, dans le cas de l'état informationnel, ces conditions sont toujours satisfaites puisqu'une relation informationnelle est définie comme une relation de covariation nomique. On peut renforcer ce premier critère par l'ajout d'une contrainte supplémentaire: pour qu'un état puisse être considéré comme représentationnel, il faut qu'il ait des conditions de correction et que ces conditions puissent être définies de manière à laisser ouverte la possibilité de méprise.

Le second critère proposé par Bermúdez est un critère d'utilité explicative. En d'autres termes, il n'est légitime de donner des explications qui font appel à des états représentationnels (des explications intentionnelles) que pour autant que ce qui est ainsi expliqué ne pourrait l'être par des explications non-intentionnelles. Ainsi, il paraît superflu d'avoir recours à des explications intentionnelles de comportements lorsqu'il existe des relations invariantes entre les entrées sensorielles et ces comportements. En revanche, de telles explications paraissent utiles pour des comportements plastiques et flexibles qui témoignent d'interactions complexes entre les entrées sensorielles et les états internes de l'organisme.

Pour que les états représentationnels jouent un rôle dans l'explication des comportements flexibles, il faut qu'ils soient susceptibles d'intégration cognitive. C'est là le troisième critère proposé par Bermúdez qui distingue en outre deux aspects de l'intégration cognitive. Premièrement, la notion d'intégration cognitive introduit l'idée d'une interaction entre états représentationnels, ce qui implique l'existence d'interfaces entre états représentationnels. Deuxièmement, le fait qu'un organisme qui représente son environnement soit en mesure de détecter des similitudes entre situations et d'exploiter celles-ci afin de mieux adapter son comportement constitue également une forme d'intégration cognitive. 
Pour que ces formes d'intégration cognitive soient possibles, il faut que les états représentationnels soient structurés et manifestent une forme de compositionnalité permettant la reconnaissance de similitudes partielles et au moins certaines formes primitives d'inférence (proto-inférences). La compositionnalité constitue ainsi le quatrième critère proposé par Bermúdez.

Ces quatre critères sont censés valoir pour tout état doté d'un contenu intentionnel, que celui-ci soit conceptuel ou non. On peut penser que l'attribution à un état d'un contenu conceptuel demande que certaines contraintes supplémentaires soient satisfaites. De ce point de vue, on notera que tant l'intégration cognitive que la compositionnalité sont susceptibles de degrés. Ce qui pourrait au moins en partie distinguer les contenus conceptuels des contenus non-conceptuels est le fait que les premiers sont soumis à des contraintes plus fortes de compositionnalité et d'intégration cognitive. Ainsi la Contrainte de Généralité que propose Evans (1982) pour les concepts peut-elle être considérée comme une contrainte forte de compositionnalité; tandis que l'isotropie et la quinicité qui constituent pour Fodor (1983) les marques distinctives des systèmes centraux conceptuels paraissent impliquer une forme très forte d'intégration cognitive. Il nous reste maintenant à voir s'il est ou non possible de donner satisfasse aux critères de Bermúdez et rende compte de la phénoménologie de ces expériences. Je commencerai pour ce faire par examiner certaines propositions avancées par Peacocke.

\section{Structure des contenus perceptifs}

Les quatre critères proposés par Bermúdez ne sont pas indépendants. La compositionnalité est requise pour l'intégration cognitive et l'intégration cognitive requise pour l'utilité explicative. La forme de compositionnalité d'un type de représentation est associée aux opérations que l'on peut appliquer à ses constituants. Les opérations possibles sont à leur tour déterminées par la manière dont ces contenus sont structurés. C'est donc en nous intéressant à la structure des contenus perceptifs que nous serons en mesure d'évaluer si ces contenus satisfont ou non aux critères de Bermúdez.

\subsection{Les contenus scénarios}

Peacocke (1992) distingue deux niveaux ou couches de contenu non-conceptuel des expériences perceptives. Le premier niveau correspond à ce qu'il appelle le contenu scénario. Les scénarios sont des types spatiaux qui spécifient la manière dont l'espace qui entoure le 
sujet est rempli. La spécification d'un scénario ou type spatial opère en deux temps. On doit tout d'abord spécifier une origine et un ensemble d'axes définis relativement au corps du sujet et à ses propriétés. Dans un deuxième temps on spécifie un mode de remplissage de l'espace autour de l'origine en indiquant pour chaque point identifié par sa direction et sa distance relativement à l'origine, s'il y a ou non une surface en ce point et si oui quelle est sa texture, sa couleur, sa saturation, sa brillance, son degré de solidité, son orientation et ainsi de suite. Un contenu scénario est dit positionné lorsqu'on assigne à son origine et à ses axes une position et des directions dans le monde et lorsqu'on lui assigne un temps.

On notera que, comme le fait remarquer Peacocke, il n'est nullement requis que les ressources conceptuelles utilisées pour spécifier un type spatial soient attribuées au sujet percevant ni que ce type spatial lui-même soit construit à partir de concepts. La notion de contenu scénario est donc parfaitement compatible avec l'idée que le contenu perceptif est non conceptuel. Nous avons considéré tout à l'heure trois propriétés importantes de l'expérience perceptive: sa finesse de grain, sa richesse informationnelle et son caractère perspectival. Le caractère perspectival de l'expérience perceptive apparaît ici comme une conséquence directe de la structure des scénarios et du fait qu'ils sont organisés en fonction d'une origine et d'un système d'axes ancrés sur le corps du sujet. Cette structure permet également de rendre compte de la richesse informationnelle et de la finesse de grain de la perception puisque pour chaque point du scénario une valeur particulière est spécifiée pour chaque dimension qualitative pertinente et que ces valeurs sont données simultanément pour tous les points du scénario. Les contenus scénarios rendent ainsi compte de certains aspects importants de la phénoménologie perceptive. Qu'est est-il des critères de Bermúdez? Les contenus scénarios sont-ils des contenus représentationnels? Tout d'abord, comme le souligne Peacocke, les contenus scénarios ont des conditions de correction que l'on peut énoncer ainsi: "Le contenu donné par le scénario positionné est correct si la scène au lieu assigné tombe sous la scénario au temps assigné lorsque le scénario y est positionné en accord avec les directions assignées" (1992: 65). Certaines remarques de Peacocke sur l'amodalité suggèrent également que les contenus scénarios satisfont au critère d'intégration cognitive. Peacocke suggère en effet que dans la mesure où les contenus d'autres modalités sensorielles sont également structurés par un système d'origine et d'axes ancrés sur le corps, il doit être possible d'intégrer ces contenus en un contenu amodal sur la base de correspondances établies entre les différents systèmes d'origine et d'axes. En outre, toute une série d'opérations exploitant la structure donnée par le système d'origine et d'axes peuvent en principe être définies et permettre, par exemple, la détection de certaines symétries globales dans un scénario ou la comparaison de plusieurs 
scénarios et la détection de similitudes entre ceux-ci. Enfin, on peut étendre les remarques de Peacocke sur l'amodalité au-delà des systèmes perceptifs. Le système moteur exploite également des référentiels égocentriques. La mise en correspondance des référentiels égocentriques perceptifs et moteurs permet de rendre compte de certaines formes d'intégration entre perception et action, telles que la navigation dans l'environnement ou les comportements d'atteinte. La structure des scénarios autorise en principe toute une série d'autres opérations sur les contenus. Cette mise en correspondance des référentiels perceptifs et moteurs rend notamment possibles certaines proto-inférences sur les conséquences perceptives de nos déplacements dans l'environnement.

Les contenus scénarios ont toutefois un pouvoir explicatif limité. Certaines de ces limitations sont d'ordre phénoménologique. Notre expérience perceptive ordinaire n'est pas pointilliste. Par la vision, nous avons l'expérience d'un monde organisé en objets tridimensionnels qui persistent à travers le temps et qui, pour certains, se meuvent dans l'espace et non l'expérience d'une tempête de confettis. Corrélativement, un grand nombre de nos proto-inférences perceptives concernent ces objets, leur comportement et leurs relations mutuelles. En outre, nos interactions avec l'environnement ne se limitent pas à des déplacements, nous interagissons avec des objets que nous manipulons de mille manières. Pour rendre compte de ces aspects de l'expérience visuelle et de ces formes supplémentaires d'intégration cognitive et de proto-inférences nous devons aller au-delà des contenus scénarios.

\subsection{Les contenus protopropositionnels}

Peacocke lui-même propose l'existence d'un second niveau ou couche de contenu nonconceptuel qu'il appelle contenu propositionnel et qu'il caractérise de la manière suivante:

Je suggère que l'expérience perceptive a une seconde couche de contenu nonconceptuel. Les contenus qui appartiennent à cette couche ne peuvent être identifiés aux contenus scénarios, mais ils sont aussi distincts des contenus conceptuels. Ces contenus supplémentaires, je les appelle protopropositions. Ces protopropositions sont évaluables comme vraies ou fausses. Une protoproposition contient un ou plusieurs individus, ainsi qu'une propriété ou relation. Lorsqu'une protoproposition fait partie du contenu représentationnel d'une expérience, l'expérience représente la propriété ou relation comme s'appliquant à l'individu ou aux individus qu'elle contient également. Je parle de protopropositions plutôt que de protopensées parce que les protopropositions contiennent des objets, propriétés et relations plutôt que des concepts de ceux-ci. Je parle de protopropositions parce que dans cette approche ce n'est pas le fait d'être fixé par un contenu conceptuel que posséderait l'expérience qui détermine qu'elles font partie de son contenu. Ces protopropositions qui font partie du contenu 
représentationnel de l'expérience visuelle ordinaire des êtres humains contiennent des propriétés et relations telles que carré, courbe, parallèle à, équidistant de, de même forme que et symétrique. On peut représenter ces propriétés et relations comme s'appliquant à des lieux, des lignes ou des régions d'un scénario positionné ou à des objets perçus comme situés en ces lieux. (Peacocke, 1992: 77).

Peacocke souligne l'utilité explicative de ce niveau de contenu. Le contenu protopropositionnel joue, selon lui, un rôle important dans la mémoire perceptive. Il encode l'information perceptive à un niveau plus abstrait que le contenu scénario et facilite la mémorisation et la reconnaissance des formes visuelles. Il permet également de décrire de manière satisfaisante l'expérience d'objets ou de figures impossibles tels qu'on les rencontre par exemple dans les dessins d'Escher. Le contenu protopropositionnel d'une telle expérience pourrait par exemple inclure que $x$ est au dessus de $y$, que $y$ est au dessus de $z$ et que $z$ est au dessus de $x$. C'est également à ce niveau que l'on peut, selon Peacocke, rendre compte de la différence entre l'expérience d'un objet comme un carré et l'expérience de ce même objet comme un losange régulier. Le contenu représentationnel de la première expérience comprend la protoproposition que la figure est symétrique par rapport aux bissectrices des côtés, celui de la seconde expérience la proposition que la figure est symétrique par rapport aux bissectrices des angles. La différence entre les deux expériences apparaît ainsi comme une différence au niveau de leur contenu protopropositionnel. Enfin, Peacocke suggère que le contenu propositionnel constitue une base pour l'ancrage des concepts observationnels qui permet de rendre compte de manière non-circulaire de leur maîtrise.

La caractérisation que propose Peacocke des contenus proto-propositionnels est beaucoup plus schématique que celle qu'il donne des contenus-scénarios. En particulier, il n'explique pas en quoi consiste le fait de représenter non-conceptuellement des objets, propriétés et relations, quel est le mode précis de structuration qui caractérise ces contenus protopropositionnels et comment ils s'articulent aux contenus scénarios. Ainsi que lui-même l'admet, une théorie substantielle des contenus protopropositionnels et de la manière dont ils sont représentés mentalement est encore à venir. Des travaux empiriques récents sur la structuration des scènes visuelles et sur l'attention perceptive suggèrent un certain nombre de pistes quant à la manière dont les contenus protopropositionnels s'élaborent et sont structurés. Je consacrerai la dernière partie de cet article à l'exploration de certaines de ces pistes. Je me concentrerai plus particulièrement sur la question de la perception des objets et de leur représentation perceptive non-conceptuelle. 


\subsection{Le défi des objets}

Selon une conception classique, longtemps dominante tant chez les philosophes que chez les psychologues, l'organisation d'une scène visuelle en objets dotés de propriétés spécifiques ou entretenant les uns avec les autres des relations données fait nécessairement intervenir des processus de conceptualisation. Ainsi, cette position était encore soutenue récemment par la psychologue Elizabeth Spelke qui affirmait que: «Les systèmes perceptifs n'organisent pas le monde en unités... Le découpage du monde en choses nous renvoie à ce qui pourrait être l'essence de la pensée et ce qui la distingue essentiellement de la perception. Les systèmes perceptifs nous livrent la connaissance d'un arrangement continu de surfaces » (Spelke, 1988: 229). Comment est-il possible de soutenir, contre cette conception, que la perception comporte une couche de contenu protopropositionnel, non-conceptuel, mettant en jeu des objets ainsi que leurs propriétés et relations? Des travaux récents, portant sur la segmentation des scènes visuelles et sur l'attention visuelle, ont conduit à remettre en cause la conception traditionnelle des relations entre perception et pensée conceptuelle et ont permis de préciser en quel sens nous pouvons percevoir une scène comme organisée en objets sans que cette perception présuppose l'intervention de processus de conceptualisation. Dans la littérature sur l'attention visuelle, la question de la nature des unités de sélection attentionnelle a occupé une place centrale au cours de la dernière décennie. L'importance de cette question pour l'élucidation des relations entre perception et conception tient à ce que les unités élémentaires de sélection attentionnelle sont, par définition, des unités qui ont été constituées pré-attentionnellement. Cette organisation pré-attentionnelle du champ visuel est le fait de mécanismes qui sont modulaires, ascendants et qui opèrent automatiquement. L'étude des unités de sélection attentionnelle permet donc de mettre en évidence certains aspects de l'organisation pré-conceptuelle du champ visuel. Les théories classiques caractérisent l'attention en termes spatiaux et considèrent que les unités de sélection sont des régions spatiales du champ visuel (Treisman et Gelade, 1980; Johnston et Dark, 1986). Des modèles plus récents suggèrent que, dans certains cas au moins, les unités de sélection sont des objets discrets. L'attention visuelle ne serait donc pas nécessairement spatiale mais pourrait être objectale. Si l'attention objectale existe, il existe des 'objets' visuels préattentionnels et la segmentation d'une scène en objets ne fait pas nécessairement intervenir de processus de conceptualisation. Je voudrais brièvement présenter quelques-unes des données récentes en 
faveur de l'attention objectale et tenter de préciser ce à quoi correspond la notion d'objet ou de proto-objet visuel. $^{4}$

Premièrement, un certain nombre d'études sur l'attention divisée — où des sujets doivent prêter simultanément attention à au moins deux aspects ou propriétés d'une scène visuelle — ont montré que les performances de ceux-ci sont meilleures lorsqu'il s'agit de propriétés d'un même objet et suggèrent ce faisant que les objets peuvent constituer des unités de sélection attentionnelle. Ainsi, dans l'étude pionnière de Duncan (1984) on présentait brièvement à des sujets des stimuli composés d'une boîte à laquelle était superposée une ligne. La boîte pouvait être carrée ou rectangulaire et comporter une ouverture sur la droite ou sur la gauche. La ligne pouvait être formée de points ou de tirets et était légèrement oblique par rapport à la verticale, penchant soit vers la droite soit vers la gauche (voir Figure $1)$.

Insérer Figure 1 à peu près ici

A chaque présentation on demandait aux sujets de juger deux de ces propriétés. Certains étaient interrogés sur deux propriétés du même objet (par exemple, forme et côté d'ouverture de la boîte); d'autres sur une propriété de chaque objet (par exemple, forme de la boîte et orientation de la ligne). On observe de meilleures performances chez les sujets interrogés sur deux propriétés du même objet. Il paraît difficile de rendre compte de ces résultats dans le cadre d'une théorie de l'attention spatiale dans la mesure où les deux objets sont superposés. Deuxièmement des études sur la diffusion automatique de l'attention montrent que cette diffusion est souvent moins efficace entre deux objets qu'à l'intérieur d'un même objet, même lorsque la séparation spatiale est la même (Duncan, 1993a, 1993b ; Baylis et Driver, 1993 ; Egly, Driver et Rafal, 1994). Ainsi, dans l'expérience de Egly, Driver, et Rafal (1994), on présente à un sujet deux barres verticales (voir Figure 2a). Il doit détecter une diminution de luminance à une extrémité de l'une des deux barres présentées. On lui donne sur l'extrémité en question un indice valide dans $75 \%$ des cas. On observe que lorsque l'indice n'est pas valide, les sujets détectent le changement de luminance plus rapidement lorsqu'il est situé à l'autre extrémité de la même barre plutôt que sur une extrémité équidistante de l'autre barre. En outre on a pu montrer à partir de ce paradigme expérimental (Moore, Yantis et Vaughan,

\footnotetext{
${ }^{4}$ Pour une revue détaillée de ces données, voir Scholl (2001).
} 
1998) que les unités de sélection prennent en compte l'occlusion, le même effet étant présent avec des stimuli tels que celui de la Figure $2 b$.

Insérer Figure 2 à peu près ici

Certaines des données les plus pertinentes pour notre propos sont issues de travaux sur les pathologies de l'attention, notamment l'héminégligence et le syndrome de Balint. L'héminégligence est un déficit attentionnel caractérisé par une incapacité pour un sujet, à la suite d'une lésion unilatérale du cortex pariétal postérieur, à détecter des signaux ou à percevoir des objets ou parties d'objets situés dans la partie contralésionnelle du champ visuel, le plus souvent l'hémichamp gauche. L'héminégligence est souvent définie comme un trouble de l'attention spatiale. Toutefois, des données récentes montrent que ce déficit ne concerne pas toujours la partie gauche de l'espace visuel définie relativement à un référentiel égocentrique mais peut concerner aussi la partie gauche d'objets ou de groupements d'objets. On peut en donner une première illustration dans une tâche de reproduction de dessins par des sujets héminégligents. Lorsque le dessin à reproduire comporte un seul objet, ces sujets n'en reproduisent que la partie gauche. En revanche, lorsque le dessin comporte deux objets côte à côte, les sujets ne dessinent pas l'objet de droite en négligeant celui de gauche mais reproduisent la partie droite de chacun des deux objets.

En outre, comme le montrent plusieurs études, l'héminégligence peut être largement affectée par des processus préattentionnels de segmentation et de ségrégation entre figure et fond. Ainsi, Driver, Baylis et Rafal (1992) ont montré à un patient héminégligent des figures vert vif sur un fond rouge pâle. On lui demandait de mémoriser la forme du contour séparant le vert du rouge et ensuite de juger si ce contour correspondait ou non à un contour-témoin. Lorsque le contour était présenté dans l'hémichamp gauche mais correspondant à la partie droite de l'objet, les jugements du sujet étaient corrects à $95 \%$, mais le pourcentage de réponses correctes tombait à $50 \%$ lorsque le contour était présenté dans l'hémichamp droit mais correspondait à la partie gauche de l'objet. Driver, Baylis et Rafal (1992) ont également montré que des patients héminégligents sont capables d'opérer une segmentation entre figure et fond sur la base des propriétés de symétrie des régions alors même qu'ils ne perçoivent pas consciemment cette symétrie. D'autres études réalisées sur des patients héminégligents montrent de manière particulièrement frappante que l'attention exploite des cadres de référence intrinsèques centrés sur des objets. Behrmann et Tipper (1994) ont mesuré le temps nécessaire à des patients héminégligents pour détecter des cibles apparaissant soit sur la partie 
droite, soit sur la partie gauche d'un objet en forme d'haltère (Figure 3). De manière prévisible, ils leur faut plus de temps pour détecter les cibles apparaissant sur la gauche. Toutefois, lorsque les expérimentateurs font subir à l'haltère, sous les yeux des sujets, une rotation à 180 degrés qui inverse ses deux côtés, les temps de réaction s'inversent, les patients mettant alors plus de temps à détecter les cibles présentées sur la partie située à droite.

Insérer Figure 3 à peu près ici

Il est crucial de noter que ce résultat n'est obtenu que lorsque l'on a affaire à un objet unique. Si la barre centrale connectant les deux disques est supprimée, l'inversion des performances n'a plus lieu. Cette expérience suggère qu'un cadre de référence intrinsèque centré sur l'haltère est défini automatiquement et que, lorsque l'objet subit une rotation, ce cadre se déplace avec lui. Les mêmes chercheurs ont réalisé récemment une expérience encore plus étonnante (Behrmann et Tipper, 1999) montrant que l'attention peut utiliser simultanément plusieurs cadres de référence. Cette expérience reprend le dispositif précédent mais ajoute à l'haltère deux carrés stationnaires, l'un positionné sur la droite, l'autre sur la gauche. Les cibles à détecter peuvent maintenant apparaître dans l'un des carrés ou dans l'un des disques de l'haltère. Avant la rotation de l'haltère, les cibles qui apparaissent dans le carré ou le disque de droite sont détectées plus rapidement que les autres. Toutefois, après rotation 180 degrés de l'haltère, ce sont les cibles présentées dans le disque maintenant situé à gauche et celles présentées dans le carré de droite qui sont détectées le plus rapidement. L'attention peut donc non seulement exploiter plusieurs cadres de référence, mais elle peut encore les exploiter simultanément.

Le syndrome de Balint fournit également des données en faveur de l'attention objectale. Ce syndrome est consécutif à des lésions bilatérales du cortex pariétal supérieur. Il est caractérisé par l'apraxie oculaire — incapacité à déplacer la fixation d'un objet sur un autre la simultanagnosie — incapacité à percevoir plus d'un objet à la fois —, la désorientation spatiale - incapacité à localiser correctement les objets et à s'orienter vers eux — et par l'ataxie optique - incapacité à atteindre un objet dans l'espace et à s'en saisir. Un sujet souffrant de ce syndrome sera incapable de percevoir plus d'un objet à la fois, même lorsque les objets sont superposés. Ainsi, Driver et Baylis (1998) rapportent le cas d'un patient qui pouvait voir les yeux du médecin mais non ses lunettes ou bien inversement voir ses lunettes mais non ses yeux. En outre, comme le rapportent également Driver et al. (1997), des patients qui ne peuvent voir qu'un cercle lorsqu'on leur en présente deux simultanément, deviennent 
capables de les voir l'un et l'autre lorsqu'ils sont reliés par une barre et forment un objet unique. De tels faits sont difficiles à expliquer dans le cadre d'un modèle purement spatial de l'attention mais on peut aisément en rendre compte dans le cadre d'une théorie objectale. Le syndrome de Balint a également pour intérêt de démontrer l'existence d'une dissociation entre la capacité à percevoir un objet et la capacité à le localiser dans l'espace et à agir sur lui. On peut décrire cette situation en disant que les patients souffrant de ce syndrome restent capables de construire un cadre de référence centré sur un objet mais ont perdu la capacité de mettre ce cadre de référence en relation avec un cadre de référence égocentrique global.

Ces données expérimentales sur l'attention et ses déficits dans certaines pathologies tendent à corroborer les thèses suivantes. Premièrement, l'attention n'est pas toujours spatiale mais peut aussi être objectale. Deuxièmement, l'attention objectale fait intervenir un cadre de référence intrinsèque, centré sur un objet (ou un groupe d'objets) et solidaire de ses déplacements. Troisièmement, la segmentation d'une scène en proto-objets et la ségrégation entre figure et fond peuvent être réalisées par des processus préattentionnels, l'attention intervenant à des étapes ultérieures et opérant sur les proto-objets générés par ces processus. Quatrièmement, les données sur l'attention objectale dans l'héminégligence suggèrent également que l'attention joue un rôle essentiel non pas tant dans la segmentation d'une scène en objets que dans la perception consciente de ces objets. Enfin, les données sur le syndrome de Balint suggèrent que l'attention pourrait aussi jouer un rôle dans le liage des cadres de référence.

Ces données posent aussi la question de la nature des proto-objets préattentionnels et des principes qui gouvernent la segmentation préattentionnelle des scènes visuelles. Selon le modèle développé par Palmer et Rock (Palmer et Rock 1994; Palmer, 1977, 1978, 1982, 1999, Rock, 1995), le principe fondamental de segmentation des scènes visuelles est un principe de connectivité uniforme, autrement dit, la tendance à percevoir des régions connectées de propriétés uniformes (luminance, couleur, texture, mouvement, disparité) comme des unités de base de l'organisation perceptive. Une fois opérée cette première analyse en régions, des principes d'organisation supplémentaires peuvent s'appliquer. Une deuxième étape fait intervenir les principes d'organisation figure/fond qui opèrent une classification des régions segmentées en figures et fond sur la base de critères tels que l'inclusion spatiale (surroundedness), la taille, l'orientation, le contraste, la symétrie, la convexité et le parallélisme. Selon Palmer, les régions désignées comme figures vont ensuite constituer les premières unités d'organisation perceptive et les unités d'entrée pour des opérations supplémentaires de groupement qui organiseront ces éléments en unités d'ordre supérieur 
ainsi que pour des opérations d'analyse en parties qui, inversement, les subdiviseront en unités subordonnées. Palmer et Rock insistent également sur le rôle des cadres de référence dans la structuration des objets et de leurs parties comme dans celle des groupes d'objets. Ils soulignent que, de toutes les propriétés perceptives des objets, la forme est de loin la plus importante car la plus informative. Ils soutiennent que la perception des formes fait intervenir des cadres de référence perceptifs au sens où la forme est une description relativement à un cadre de référence donné. En outre, ils affirment que le système perceptif n'utilise pas un cadre de référence général unique pour décrire la forme des objets. Au contraire, chaque objet est décrit à partir d'un cadre de référence spécifique choisi sur la base des propriétés intrinsèques de l'objet. Les facteurs importants pour la détermination d'un cadre de référence comprennent les axes de symétrie réflexive, les axes d'élongation, l'orientation des contours, l'orientation de la texture, l'orientation contextuelle, l'orientation gravitationnelle et la direction du mouvement. Certains de ces facteurs correspondent à des propriétés intrinsèques de l'objet tandis que d'autres, tels l'orientation contextuelle et l'orientation gravitationnelle sont extrinsèques. Palmer et Rock suggèrent que les propriétés extrinsèques jouent un rôle important lorsque la structure interne de l'objet est faible, comme dans les objets informes, ou ambiguë, comme dans les triangles équilatéraux. On notera en outre un large recoupement entre les propriétés exploitées pour définir l'organisation figure/fond et les propriétés utilisées pour la définition d'un cadre de référence intrinsèque, ce qui suggère que les deux opérations sont étroitement liées. Les données sur l'héminégligence montrent que les opérations de segmentation en régions, d'organisation figure/fond et de définition de cadres de référence intrinsèques peuvent être le fait de processus préattentionnels de type ascendant.

L'objectif de cette brève excursion dans le domaine de la psychologie de la perception et de la psychologie de l'attention était de nous permettre de préciser la manière dont les contenus protopropositionnels s'élaborent et sont structurés et ainsi d'en mieux cerner l'utilité explicative. Ces travaux montrent l'existence d'un niveau d'organisation perceptive où la scène visuelle est segmentée en proto-objets. Ces proto-objets sont définis sur la base de propriétés telles que la connectivité uniforme, la convexité, la symétrie. Cette segmentation résulte de l'opération de processus de segmentation pré-attentionnels et pré-conceptuels. Les protoobjets ainsi définis sont structurés par des cadres de référence intrinsèques, exploitant leurs propriétés (axes, symétries). Les divers traits et propriétés de l'objet sont ensuite encodés relativement à ce cadre de référence. Ce mode de structuration peut opérer récursivement, soit au niveau subordonné par une analyse d'un objet en ses parties, soit au niveau surordonné par des groupements. Les données sur l'attention objectale mettent en outre en évidence la 
possibilité de dissociations entre la perception d'un objet et sa localisation spatiale (héminégligence et syndrome de Balint) et donc d'une part une relative indépendance des modes de structuration qui font intervenir cadres de référence égocentriques et cadres de référence intrinsèques et d'autre part l'existence d'un problème de liage des cadres de référence.

Je voudrais pour terminer revenir une dernière fois sur les critères de Bermúdez et sur les avantages explicatifs que présente ce niveau de contenu. Tout d'abord, une expérience perceptive dotée de contenu protopropositionnel nous présente une scène visuelle structurée en objets dotés chacun d'une forme spécifique caractérisée relativement à un cadre de référence intrinsèque. Ce processus d'organisation opère en outre de manière récursive, les unités élémentaires que sont les objets organisés dans des structures plus importantes (groupements) ou analysés en leurs parties. Une expérience dotée de ce type de contenu a donc des conditions de correction qui vont au-delà des conditions attachées aux contenus scénarios. Le contenu protopropositionnel d'une expérience est correct si et seulement si la disposition des objets dans la scène visuelle aux temps et lieu assignés est compatible avec le mode de segmentation entre figures et fond que présente le contenu, si les objets dans la scène ont les propriétés et ont entre eux les relations spatiales que le contenu les présente comme possédant.

Ce niveau de contenu a également pour caractéristique de manifester une forme d'intensionnalité qui n'est pas encore présente dans les scénarios. Cette forme d'intensionnalité est illustrée par l'exemple de la forme que l'on peut percevoir comme un carré ou comme un losange. Selon Peacocke, le contenu représentationnel de la première expérience comprend la protoproposition que la figure est symétrique par rapport aux bissectrices des côtés, celui de la seconde expérience la proposition que la figure est symétrique par rapport aux bissectrices des angles. Nous pouvons reformuler l'idée de Peacocke en disant que cette forme admet deux descriptions structurelles faisant intervenir des cadres de références différents: l'une utilise un cadre de référence intrinsèque prenant pour axes les bissectrices des côtés, l'autre un cadre de référence intrinsèque prenant pour axes les bissectrices des angles. L'avantage de cette formulation qui fait intervenir la notion de cadre de référence est d'avoir une portée plus large, puisqu'elle s'applique à tous les objets ou groupements d'objets qui sont structurellement ambigus.

Sur le plan de l'utilité explicative, ce niveau de contenu qui fait intervenir un mode de structuration plus élaboré de l'expérience perceptive permet des formes d'intégration cognitive et des proto-inférences nouvelles par rapport à celles qu'autorisent les contenus scénarios. 
L'intégration cognitive consiste pour une part en la capacité d'une créature à détecter des similitudes partielles entre les situations qu'elle rencontre. Nous avons vu que l'exploitation de la structure des scénarios rend possible la détection de certaines de ces similitudes. Toutefois, la détection de similitudes par l'exploitation de la structure des scénarios est soumise à de sévères restrictions. Les similitudes détectées sont pour l'essentiel des similitudes relativement globales qui dépendent d'opérations de rotation et/ou de translation du système de coordonnées. Lorsque plusieurs cadres de référence égocentriques et intrinsèques et pour certains éventuellement enchâssés, sont utilisés pour structurer une expérience perceptive, de nouvelles similitudes peuvent être détectées. Par exemple, l'utilisation de cadres de référence centrés sur les objets permet la détection d'équivalence de formes qui ne seraient pas détectables sur la seule base des contenus scénarios. Plus généralement, l'utilisation de ces cadres de références permet la reconnaissance de similitudes indépendamment du point de vue et leur enchâssement la détection de similitudes à des échelles différentes. On peut par exemple voir que l'organisation générale de deux scènes est la même en dépit du fait que les unités ainsi organisées sont différentes.

Un autre aspect de l'intégration cognitive concerne les interactions des états perceptifs avec d'autres types d'états représentationnels. Là encore, l'enrichissement structurel qu'apporte l'utilisation simultanée de plusieurs cadres de référence permet d'aller au-delà de ce qui est possible sur la base des contenus scénarios. En premier lieu, la construction de contenus amodaux beaucoup plus riches devient possible. Par exemple, à supposer que d'autres modalités que la modalité visuelle fasse également intervenir des cadres de références intrinsèques, les objets présentés sous une modalité peuvent être comparés avec des objets présentés sous une autre modalité et leur identité de forme éventuellement détectée. Ainsi, on devrait être capable de détecter l'équivalence entre un cube vu et un cube exploré par le toucher qui n'occupent pas la même position dans l'espace — ce qui n'est pas possible pour les contenus amodaux fondés sur les contenus scénarios. Cette structuration par des cadres de références non-égocentriques permet également la construction de cartes allocentriques de l'environnement, autrement dit de cartes qui définissent les relations spatiales entre objets et traits de l'environnement indépendamment de la position de l'observateur en exploitant des cadres de référence centrés sur des objets ou des traits de l'environnement. De telles cartes ont une stabilité qui fait défaut aux représentations égocentriques: elles n'ont pas à être mises à jour à chaque fois que l'observateur se déplace. Elles permettent ainsi d'accroître les capacités de navigation et de repérage de l'agent dans son environnement. Une autre conséquence de cette structuration de la scène visuelle en objets est d'autoriser des proto-inférences sur le 
comportement des objets et non simplement des proto-inférences sur les conséquences de notre propre comportement sur le contenu de notre expérience perceptive. Il est bien sûr trivial de dire que l'expérience perceptive ne nous permettrait pas de faire des inférences sur le comportement des objets si elle ne nous présentait pas des objets. Il est en revanche intéressant de remarquer que les principes que le système visuel utilise pour la segmentation d'une scène en objets, pour le groupement d'objets ou pour l'analyse de leurs parties peuvent aussi être exploités pour faire des proto-inférences sur leur comportement. Ainsi, nous nous attendons à ce que les objets qui ont été définis en utilisant le principe de connectivité uniforme se déplacent "en bloc". Cette attente est également présente dans une certaine mesure pour les objets qui ont été groupés ensemble même si le principe qui a présidé à leur groupement n'est pas le principe de mouvement commun. On doit encore souligner le fait que ce mode de structuration permet un encodage mnésique plus efficace et plus économique de l'information visuelle qu'un encodage fondé sur la structure des scénarios. Enfin, l'information perceptive ainsi structurée peut être exploitée par le système moteur pour des comportements plus complexes que les comportements d'atteinte ou de navigation. Une grande partie de nos comportements moteurs font intervenir la manipulation d'objets et les manipulations auxquelles un objet peut être soumis dépendent en grande partie de sa forme intrinsèque.

\section{Conclusion}

Mon objectif était d'apporter une contribution à la défense de la thèse selon laquelle l'expérience perceptive est en partie non-conceptuelle. Après avoir rappelé un certain nombre d'arguments négatifs visant à montrer que le contenu de la perception ne saurait être purement conceptuel, j'ai tenté de prolonger l'essai de caractérisation positive du contenu nonconceptuel de la perception qu'a proposé Peacocke et en particulier de préciser la notion de contenu protopropositionnel qu'il introduit. Dans sa discussion $\mathrm{du}$ contenu protopropositionnel, Peacocke met surtout l'accent sur les éléments de ce contenu: individus, propriétés et relations. J'ai essayé de proposer une perspective complémentaire en mettant l'accent sur le mode de structuration qui rend possible que l'expérience perceptive ait ce contenu. J'ai insisté tout particulièrement sur le fait que cette structuration met en jeu des cadres de référence intrinsèques et fait intervenir simultanément plusieurs cadres de référence. J'ai souligné que cette structuration peut être le résultat de processus de traitement ascendants pré-attentionnels et qu'ainsi la structuration d'une scène en objets n'implique pas nécessairement de processus de conceptualisation. J'ai également essayé de montrer que ce niveau de contenu est bien représentationnel et satisfait aux critères de Bermúdez. 


\section{Références}

Baylis, G. C. \& Driver, J. 1993. Visual attention and objects. Journal of Experimenal Psychology, Human Perception and Performance, 3, 451-470.

Behrmann, M. \& Tipper, S. P. 1994. Object-based attentional mechanisms. In C. Umiltà and M. Moscovitch (eds), Attention and Performance XV, Cambridge, MA: MIT Press.

Behrmann, M. \& Tipper, S. P. 1999. Attention accesses multiple reference frames: evidence from unilateral neglect. Journal of Experimental Psychology: Human Perception and Performance, 25, 83-101.

Bermúdez, J. L. 1998. The paradox of self-consciousness. Cambridge, MA: MIT Press.

Brewer, B. 1999. Perception and Reason. Oxford: Oxford University Press.

Burns, E. M. \& Ward, W. D. 1977. Categorical perception - phenomenon or epiphenomenon: Evidence from experiments in the perception of musical intervals. Journal of the Acoustical Society of America, 63: 456-68.

Crane, T. 1992. The non-conceptual content of experience. In The Contents of Experience, ed. T. Crane, 136-157. Cambridge: Cambridge University Press.

Dokic, J. \& Pacherie, E. 2001. Shades and concepts. Analysis, 61, 3, 193-202.

Dretske, F. 1969. Seeing and Knowing. Chicago: The University of Chicago Press.

Dretske, F. 1981. Knowledge and the Flow of Information, Cambridge, MA: MIT Press.

Dretske, F. 1995. Naturalizing the Mind - The Jean Nicod Lectures - 1994. Paris: Editions du CNRS \& Cambridge, MA: MIT Press.

Driver, J. \& Baylis, G. C. 1998. Attention and visual object segmentation. In R. Parasuman (ed.), The Attentive Brain. Cambridge, MA: MIT Press, 299-325.

Driver, J., Baylis, G. C., \& Rafal, R. D. 1992. Preserved figure-ground segregation and symmetry perception in visual neglect. Nature, $360,73-75$.

Driver, J., Mattingley, J.B., Rorden, C., \& Davis, G. 1997. Extinction as a paradigm measure of attentional bias and restricted capacity, following brain injury. In R. Thier and H.-O. Karanth (eds), Parietal Lobe Contributions to Orientation in 3D Space, Berlin: Springer-Verlag, 401-429.

Duncan, J. 1984. Selective attention and the organization of visual information. Journal of Experimental Psychology: General, 113, 501-17.

Duncan, J. 1993a. Coordination of what and where in visual attention. Perception, 22, 12611270.

Duncan, J. 1993b. Similarity between concurrent visual discriminations: Dimensions and objects. Perception and Psychophysics, 54, 425-430.

Egly, R. Driver, J. \& Rafal, R. 1994. Shifting visual attention between objects and locations: Normality and pathology. Journal of Experimental Psychology, General, 123, 161-177.

Evans, G. 1982. The Varieties of Reference. Oxford: Clarendon Press.

Fodor, J.A. 1983. The Modularity of Mind, Cambridge, Mass.: MIT Press.

Halsey, R. M. \& Chapanis, A.1951. Number of absolute identifiable hues. Journal of the Optical Society of America, 41: 1057-8.

Hardin, C. L. 1988. Color for philosophers. Indianapolis: Hackett.

Hurvich, L. M. 1981. Color vision. Sunderland, MA: Sinauer Associates.

Johnston, W. A. \& Dark, V. J. 1986. Selective attention. Annual Review of Psychology, 37 , 43-75.

McDowell, J. 1994. Mind and World. Cambridge, MA: Harvard University Press.

Moore, C., Yantis, S. \& Vanghan, B. 1998. Object-based visual selection: evidence from perceptual completion. Psychological Science, 9, 104-110. 
Palmer, S. E. \& Rock, I. 1994. Rethinking perceptual organization: The role of uniform connectedness. Psychonomic Bulletin and Review, 1, 29-55.

Palmer, S. E. 1977, Hierarchical structure in perceptual representation. Cognitive Psychology, 9, 441-474.

Palmer, S. E. 1978. Structural aspects of visual similarity. Memory and Cognition, 6, 91-97.

Palmer, S. E. 1982. Symmetry, transformation, and the structure of perceptual systems. In J. Beck (ed.), Organization and Representation in Perception. Hillsdale, NJ: Erlbaum.

Palmer, S. E. 1999. Vision Science. Cambridge, MA: MIT Press.

Peacocke, C. 1992. A Study of Concepts. Cambridge, MA: MIT Press.

Peacocke, C. 1998. Nonconceptual content defended. Philosophy and Phenomenological Research, LVIII, 2, 381-388.

Peacocke, C. 2001. Does perception have a nonconceptual content? The Journal of Philosophy, 98, 5, 239-64.

Rock, I. 1995. Perception. New-York: Scientific American Library.

Scholl, B. J. 2001. Objects and attention: the state of the art. Cognition, 80, 1-46.

Sedivy, S. 1996. Must conceptually informed perceptual experience involve non-conceptual content? Canadian Journal of Philosophy, 26, 3, 413-31.

Spelke, E. 1988. Where perceiving ends and thinking begins: the apprehension of objects in infancy. In A. Yonas (ed.), Perceptual Development in Infancy, Hillsdale, NJ: Erlbaum, 197-234.

Treisman, A \& Gelade, G. 1980. A feature integration theory of attention. Cognitive Psychology, 12, 97-136. 

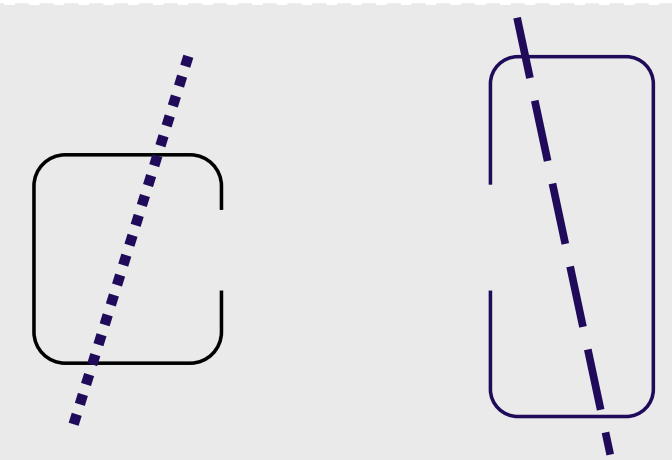

Figure 1 : Exemples de stimuli utilisés par Duncan (1984). 


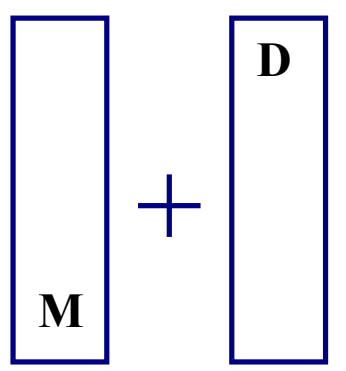

(a)

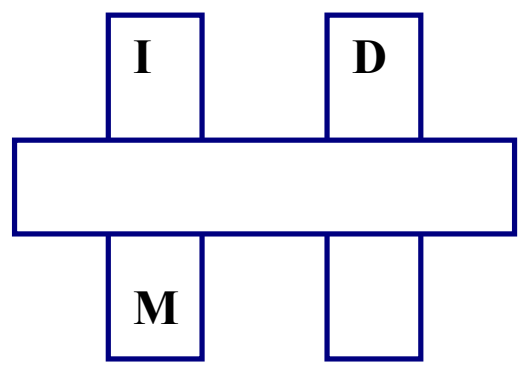

(b)
I : Indice

M: Cible sur la même barre

$\mathrm{D}$ : Cible équidistante sur l'autre barre

Figure 2 : Exemples de stimuli utilisés dans les expériences sur la diffusion automatique de l'attention; (a) Egly, Driver et Rafal (1994); (b) Moore, Yantis et Vaughan (1998). 


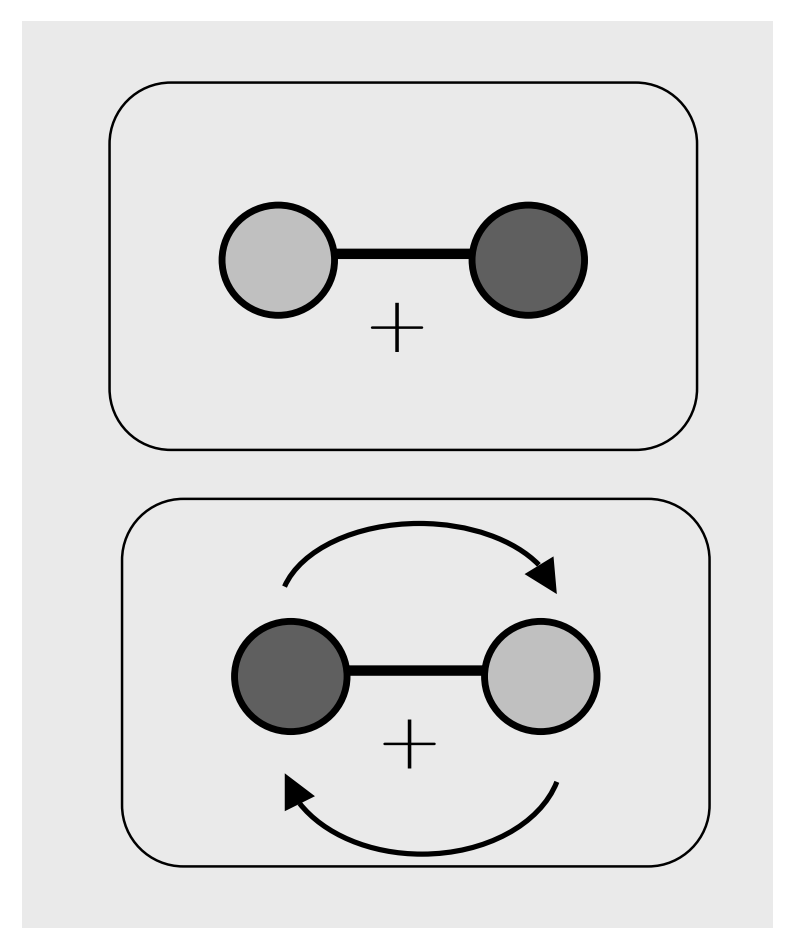

Figure 3. Avant rotation, les sujets détectent les cibles lumineuses présentées dans le disque vert (ici gris foncé) situé à droite plus rapidement que celles qui sont présentées dans le disque rouge (ici gris clair) sur la gauche; après rotation ce sont toujours les cibles présentées sur le disque vert (gris foncé) maintenant à gauche qui sont détectées le plus rapidement. D'après Behrmann et Tipper (1994). 